\title{
Optimization Algorithms for Hierarchical Problems, with Application to Nanoporous Materials: Final Technical Report (R. M. Lewis)
}

\author{
Robert Michael Lewis \\ Department of Mathematics \\ College of William and Mary \\ Williamsburg, Virginia 23185-8795. \\ rmlewi@wm . edu.
}

June 27, 2013 


\title{
Final Technical Report
}

\author{
DOE Award Number: DE-SC0001691
}

Institution: College of William and Mary

Project Title: Optimization Algorithms for Hierarchical Problems, with Application to Nanoporous Materials

Principal Investigator: Robert Michael Lewis

Research Team Members: Paul Boggs, David Gay, Stewart Griffiths, Robert Michael Lewis, Kevin Long, Stephen Nash, Robert Nilson

Reporting Period: August 15, 2009 to August 14, 2012

\section{Executive Summary}

This project investigated computational methods for optimizing the design of nanoporous materials. These materials hold promise in energy storage applications such as electrical supercapacitors and hydrogen storage systems. A significant issue for nanoporous storage devices is the design of the channel network within these materials to allow rapid diffusion while retaining storage capacity.

Nanoporous materials possess a hierarchical structure insofar as the physical behavior of flows changes with the physical length-scale one considers. For example, an end user of an energy storage device might be interested only in the bulk system, how much it can store and how quickly it can discharge. However, these macroscopic performance metrics are determined by the layout of the channel network within the storage device. Inside the channel network the flow and storage properties depend on the channel size. In large channels one can use viscous flow as a model, while in nanochannels Knudsen flow is appropriate, while there is a transition between these flow regimes for the intermediate channel widths. These flows are described by different mathematical models. In the computational design problem it is important to account for physical behavior at finer levels (e.g., Knudsen flow) to obtain accuracy at coarser levels.

There were two major research thrusts. The first involved the development of hierarchical optimization models for nanoporous materials and other energy-related applications. We sough a balance between the conflicting aims of physical fidelity and computational tractability. These models were use to determine the properties relevant to the hierarchical optimization algorithms. The second research effort was the development, analysis, and implementation of a class of hierarchical optimization algorithms, as well as the application of these algorithms to our hierarchical models. This entailed the development of flexible software that can be used to solve a variety of hierarchical optimization problems. Here is a brief summary of the team's accomplishments:

- The formulation of hierarchical models of nanoporous materials suitable for use in conjunction with computational optimization algorithms.

- The development of an multilevel optimization algorithm for constrained optimization.

- The development of an domain decomposition optimization algorithm for constrained optimization.

- The development of automatic grid refinement algorithm to transfer between length scales.

- The derivation of physically accurate procedures to adjust parameters and data between scales.

- The development of a network approximation to obtain a good initial solution.

- PDE and SDP approximation techniques to obtain a good initial solution.

- Produced a prototype implementation to test our procedures based on Sundance, a powerful system developed by Sandia National Laboratories for handling PDEs.

While our project focused on the development of optimization techniques that operate on different physical length-scales for optimizing the internal structure of nanoporous materials, the algorithms we developed can be applied to more general hierarchical design problems arising from the design of complex systems. 


\section{Overview}

This project was part of a collaborative grant involving the following researchers:

- Paul Boggs (Sandia Livermore National Laboratories)

- David Gay (AMPL Optimization LLC)

- Stewart Griffiths (Sandia Albuquerque National Laboratories)

- Robert Michael Lewis (College of William and Mary)

- Kevin Long (Texas Tech University)

- Stephen Nash (George Mason University)

- Robert Nilson (Sandia Livermore National Laboratories)

The broad research objective of the overall project was the development of multi-scale optimization approaches to aid in the design of energy storage systems. More specifically, we focused on the development of optimization methods that operate on different physical length-scales for optimizing the internal structure of nanoporous materials. Potential applications include electrical supercapacitors and hydrogen storage systems. The optimization algorithms we have developed can be applied to more general hierarchical design problems arising from the design of complex systems.

Nanoporous materials possess a hierarchical structure, and the physical behavior of flows change with the length-scale. It is important to account for physical behavior at the fine level (e.g., Knudsen flow) to obtain accuracy at coarser levels. From the perspective of using nanoporous materials in energy storage a significant issue is the fabrication of channels within these materials to allow rapid diffusion while retaining storage capacity. One goal of our research was to apply optimization methods to the design channel structure in nanoporous materials.

There are two major research thrusts. The first involves hierarchical optimization models for nanoporous materials and other energy-related applications. The models will attempt to balance the conflicting aims of model fidelity and computational tractability. We will analyze the general hierarchical model, as well as specific application models, to determine the properties that are relevant to the hierarchical optimization algorithms that we propose.

The second is the development, analysis, and implementation of a class of hierarchical optimization algorithms, as well as the application of these algorithms to our hierarchical models. A major aspect will be to develop flexible software that can be used to solve a variety of hierarchical optimization problems. Because of the large size and complexity of the optimization models, we believe that traditional optimization methods are incapable of obtaining solutions, and that it will be necessary to use these new hierarchical algorithms.

Here is a brief summary of our major accomplishments:

- The formulation of hierarchical models of nanoporous materials suitable for use in conjunction with optimization algorithms.

- The development of a convergent optimization-based multilevel algorithm for constrained optimization problems. Our algorithm allows for the first time consideration of all relevant scales in a consistent and general framework. It is the first multigrid-type algorithm capable of solving models with general equality and inequality constraints.

- The development of automatic grid refinement algorithm to facilitate movement and communication between scales.

- The derivation of physically accurate procedures to adjust parameters and data between scales.

- The development of a network approximation to obtain a good initial solution.

- Produced a prototype implementation to test our procedures based on Sundance, a powerful system for handling PDEs.

The following subsections describe these and other accomplishments in greater detail. 


\section{Model of Flow in Nanoporous Materials}

Our research uses a continuum model described by partial differential equations (PDEs) that describe the flow of gas through a nanoporous material. There is a bulk matrix that provides storage capacity, while the material includes channels that allow for more rapid movement of the gas in and out of the material. Diffusion is also possible in the bulk matrix, but at a slower rate.

The flow model assumes viscous flow in the channels and Darcy flow in the bulk. Density $\rho$ and pressure $p$ are related by the ideal gas law: $p=\rho k_{B} T$. The temperature $T$ is assumed to be constant. The resulting transport equation for the gas is a diffusion equation, with diffusion coefficients that vary depending on whether the flow is in a channel or in the bulk material.

With this model of the underlying physical system we can define the basic optimization model. Let $w$ be the set of design variables that define the size and position of the channels. Let $F(w)$ be the desired performance objective, for example, the total out-flow over a specified time interval. Let $C(w)$ represent a set of constraints, e.g., upper bounds on the charge or discharge times, or a lower bound on the total storage capacity. Then the optimization problem is

$$
\begin{array}{ll}
\underset{w}{\operatorname{minimize}} & F(w) \\
\text { subject to } & \text { transport diffusion equation for } p, \\
& \text { boundary conditions on } p, \\
& C(w) \leq 0 .
\end{array}
$$

The objective $F$ typically depends on the design variables pressure in an indirect manner, through the pressure $p$ that results from solving the transport equation given the channel network specified by $w$.

This basic optimization problem forms the basis for our optimization model. It is enhanced by defining the model in terms of multiple levels of refinement, with adjustments to the physics of the model as the size of the channels is reduced. This model corresponds to one level in our hierarchy of models. To ensure consistency between levels of the hierarchy, we conducted a mathematical analysis of the relationships between the levels. On higher levels, additional smaller-scale channels are added. Our analysis establishes relationships between the diffusivities on different levels, so that the effect of the additional channels at finer scales can be captured in the effective material properties used in the coarser scale models.

This is a time-dependent model, which could prove expensive to evaluate or optimize. In an attempt to reduce the computational cost, two members of the team, Nilson and Griffiths, developed surrogate steady-state flow models that approximate this equation. One alternative approach developed by Lewis ${ }^{1}$ approximates output functionals of the time-dependent flow field using steady-state models, while another alternative uses a semidefinite programming optimal mixing time version of the problem as an approximation. In the latter approach one minimizes the smallest eigenvalue of the steady-state diffusion operator in order to obtain an optimal bound on the time it takes to achieve steady state. Because these approaches are based on steady-state calculations, the time dependence is eliminated, which greatly reduces the computational cost.

This optimization model was used in a hierarchical framework based on finite elements. At a coarse level there are a relatively small number of larger elements, with channels along the edges of the elements. This will result in a small number of decision variables $w$ at a coarse level. The finite element is then subdivided to produce finer meshes and higher-fidelity approximations. We conducted mathematical analysis to determine appropriate ways to refine the meshes and the models. We also analyzed the change in the models between levels in the hierarchy, to construct a consistent set of models suitable for computation. As a model is coarsened some channels are eliminated, resulting in nonlinear changes to the diffusivity of the material. The resulting hierarchical model was implemented in software (see Section 4).

\section{Network Flow Models}

Typically the high-fidelity continuum models of the nanoporous materials will be expensive to evaluate and solve. For this reason we developed simpler models for the flow through the material inspired by network flow models. In this approach the physical channel network is represented by a mathematical network model

\footnotetext{
${ }^{1}$ Jointly with David Phillips, Department of Mathematics, United States Naval Academy.
} 
for which optimization is much less computationally expensive. The network-flow models are then be used to find good approximate solutions that serve as initial design the algorithms based on a continuum model.

The network-flow models are solved using greedy heuristics based on algorithms for finding spanning trees of weighted networks. These techniques are augmented with a Metropolis-Hastings random search algorithm to improve the greedy starting points. Our greedy heuristics choose a spanning tree that connects all nodes to a specified root node (the outlet, in an energy storage system). To do so, they maintain a frontier of nodes connected with the root. From among any unconnected nodes that remain, a "best" one that can be connected to the frontier is chosen, and a most attractive arc (channel)from the chosen node to a node in the frontier is added to the spanning tree. We tried several definitions of "best" and "attractive" based on physical considerations. Randomization proved useful in breaking ties in selecting nodes and arcs to add.

The heuristics used in the discrete algorithms are based on maximizing flow and improving porosity. The results have been assessed by the teams' subject-matter experts, Griffiths and Nilson, to ensure that the results are useful for initializing the more expensive algorithms that use the continuum model.

A typical result of the discrete algorithm is illustrated in Figure 1.

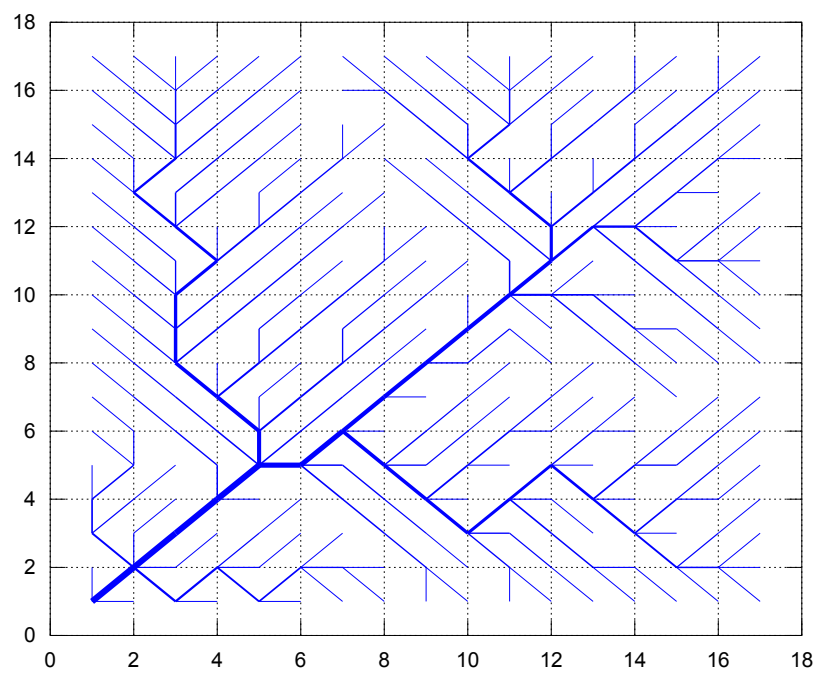

Figure 1: A channel network produced by the network flow algorithm. The outlet is in the lower left-hand corner. Note the large feeder channel along the diagonal, with increasingly smaller channels that feed it.

\section{Extensions to Sundance}

As part of this project additional capabilities were added to the Sundance PDE software system in order to integrate it with the hierarchical optimization approaches developed in this project. The Sundance package, developed by Sandia National Laboratories, is a high-level system for handling partial differential equations using high-performance parallel finite-element techniques. Sundance automatically builds the mass and stiffness matrices needed for the finite element method and provides sensitivities via the adjoint method. These powerful features make Sundance an excellent analysis tool to integrate with our optimization software.

Sundance was originally developed by a member of the research team, Kevin Long. Sundance provides a set of high-level components with which one can specify a problem and combine it with other elements (e.g., an objective function or constraints) to create useful features and procedures. The high-level nature of the components means that one need not worry about tedious and error-prone bookkeeping details. In addition to the advantage of conceptual and practical simplicity, this component-based approach allows a high degree of flexibility in the formulation, discretization, automatic differentiation, and solution of analysis and optimization problems. 
It was necessary to enhance and expand the Sundance software to handle our models of nanomaterials. There are two types of flow within the nanomaterial, flow in the channels and flows in the bulk material itself. The channel flow is confined to edges of the finite element mesh, and the original Sundance software was not capable of representing flow along these edges. As part of this project, Sundance has been modified to add the capability to define functions on edges of finite-element meshes. As a result of these developments, we are able to compute the gradients necessary to solve the model optimization problem. Another enhancements to Sundance that was added in order to implement the multilevel optimization algorithms was an automatic grid refinement feature that creates finer-scale channels as well as finer-scale bulk elements.

These enhancements of Sundance enabled the research team to develop a prototype of our multilevel optimization algorithm applied to the nanoporous material problem. An example result is illustrated in Figure 2.

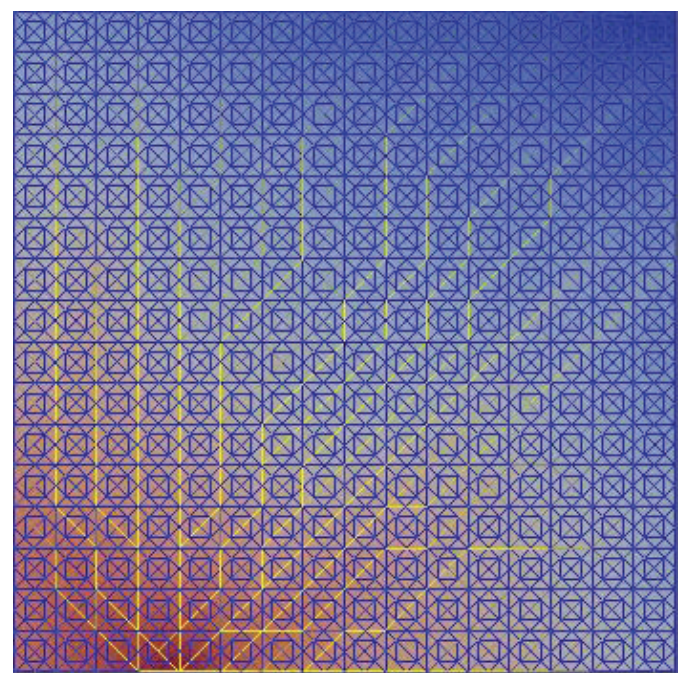

Figure 2: Optimal channel layout computed using hierarchical optimization and the continuum PDE model. The red-blue color scale of the background represents the pressure field associated with the optimal channel network.

\section{Hierarchical Algorithms for Constrained Optimization Models}

Two of the participants in the project, Lewis and Nash, earlier developed a class of multilevel, hierarchical optimization algorithms for unconstrained optimization. This earlier work provided motivation and grounding for the current research project.

As part of this research progress we developed a related multilevel optimization approach, termed MG/Opt, for the solution of the more challenging problem of constrained optimization. The approach assumes that one has a hierarchy of models, of an underlying optimization problem, ordered from fine to coarse in terms of their physical detail, and that one is interested in finding solutions at the finest level of detail. In this hierarchy of models calculations on coarser levels are less expensive, but also are of less fidelity, than calculations on finer levels. The idea of MG/Opt is to use calculations on coarser levels to accelerate the progress of the optimization on the finest level.

The new algorithms apply to optimization problems with both equality and inequality constraints. In the context of the design of nanoporous materials, the equality constraints include the differential equations describing the flow within the nanoporous material and constraints on the aggregate porosity of the material (which reflects the storage capacity). The inequality constraints include limits on the channel widths, and bounds on other material properties.

In addition to developing the algorithms, we have proven global convergence theorems for the algorithms (i.e., convergence to a Karush-Kuhn-Tucker point from an arbitrary starting point). The convergence results 
apply to a broad class of algorithms with minimal assumptions about the properties of the coarse models, and thus are expected to apply to a broad range of models of the nanoporous materials.

We also analyzed the descent properties of the algorithm, i.e., whether the coarse level correction is guaranteed to result in improvement of the fine level solution. Although additional assumptions are required to guarantee improvement, the assumptions required are likely to be satisfied by a broad range of optimization problems. Thus we not only have guarantees of convergence, but also guarantees that the hierarchical optimization approach will improve the solutions to our models.

At the finest scales, the optimization models will be very large, requiring sophisticated algorithms and computing techniques. To deal with this, PI Nash developed optimization-based domain-decomposition algorithms suitable for constrained optimization problems. These algorithms have the potential of increasing the parallelism of the optimization algorithms, and hence making the computational problem more tractable. Experimental tests with Sundance showed promising results.

In order to obtain good initial estimates of the channel widths on the finest scales, full multigrid initialization is used, making full use of the hierarchy of models.

\section{Publications and other products developed under the award}

This section reviews the products developed by the current author, PI Lewis. The following papers are a result of the project:

- Robert Michael Lewis, David Phillips, and Rui Zhang, Semidefinite covering relaxations to find graphs with maximal algebraic connectivity, in preparation.

- Robert Michael Lewis and Stephen G. Nash, Using inexact gradients in a multilevel optimization algorithm, Computational Optimization and Applications, 2013 (to appear in print, currently published online).

- Paul T. Boggs, David M. Gay, Stewart K. Griffiths, Robert Michael Lewis, Kevin R. Long, Stephen Nash and Robert H. Nilson, Optimization Algorithms for Hierarchical Problems with Application to Nanoporous Materials, SIAM Journal on Optimization, vol. 22, no. 4, 2012, pp. 285-1308.

- Stephen G. Nash and Robert Michael Lewis, Assessing the Performance of an Optimization-Based Multilevel Method, Optimization Methods and Software, vol. 26, no. 4/5, 2011, pp. 693-717.

The following conference talks by R. M. Lewis were related to this project:

- Preliminary design of nanoporous materials via semidefinite programming, SIAM Conference on Computational Science and Engineering, Boston, February 2013.

- Using Inexact Gradients in a Multilevel Optimization Algorithm, International Symposium on Mathematical Programming, Berlin, Germany, August 2012.

- Approximation and Correction Techniques for Design of a Nanoporous Material SIAM Conference on Optimization, Darmstadt, Germany, May 2011.

- Numerical experience with a multilevel optimization approach: runtime performance assessment, International Symposium on Mathematical Programming, Chicago, August 2009. 